\title{
Lattice studies of hadron physics with disconnected quark loops
}

\section{Takashi Kaneko for JLQCD Collaboration}

KEK Theory Center, High Energy Accelerator Research Organization (KEK), Tsukuba, Ibaraki 305-0801, Japan

School of High Energy Accelerator Science, The Graduate University for Advanced Studies (Sokendai), Tsukuba, Ibaraki 305-0801, Japan

E-mail: takashi.kaneko@kek.jp

\begin{abstract}
Disconnected diagrams give crucial contributions to the physics of flavor singlet hadrons and to scalar form factors of non-singlet hadrons. Naive lattice calculation of the disconnected diagrams, however, requires a huge number of fermion matrix inversions and hence a prohibitively large computational cost. In this article, we present recent studies of the flavor-singelt meson spectrum and nucleon strange quark content using the all-to-all propagator to calculate the relevant disconnected diagrams.
\end{abstract}

35th International Conference of High Energy Physics - ICHEP2010,

July 22-28, 2010

Paris France 


\section{Simulation method}

Disconnected diagrams give crucial contributions to interesting physics including the flavorsinglet meson spectrum and nucleon strange quark content. Interesting nature of the former, such as the famous $U(1)$ problem and the ideal mixing of vector mesons, has to be confirmed from first principles, and the latter is an important parameter in direct experimental searches for the dark matter. In this article, we present studies by the JLQCD Collaboration $[1,2,3]$ that use a recently proposed method, namely the all-to-all quark propagator [4].

Our gauge configurations of three-flavor QCD are generated at a lattice spacing $a \simeq 0.1 \mathrm{fm}$ using the overlap quark action, which exactly preserves chiral symmetry on the lattice. We simulate four values of degenerate up and down quark masses $m_{l}$, which cover a region of the pion mass $300 \lesssim M_{\pi}[\mathrm{GeV}] \lesssim 550 \mathrm{MeV}$, and two strange quark masses $m_{s} \simeq m_{s, \text { phys }}$ and $(5 / 4) m_{s, \text { phys }}$ with $m_{s, \text { phys }}$ the physical mass. The flavor-singlet meson spectrum is calculated on a spatial lattice volume $(1.8 \mathrm{fm})^{3}$, and we also simulate a larger volume $(2.7 \mathrm{fm})^{3}$ for the nucleon strange quark content to avoid finite volume effects. We refer readers to Ref. [5] for further details of our gauge ensembles.

It is conventional to calculate connected hadron correlators by using the so-called point-to-all quark propagator $S_{F}\left(x^{\prime}, x\right)$, which flows from a fixed lattice site $x$ to any site $x^{\prime}$ and is obtained by solving

$$
\sum_{x^{\prime}} D\left(y, x^{\prime}\right) S_{F}\left(x^{\prime}, x\right)=\delta_{y, x}
$$

where $D$ is the Dirac operator. One can naively evaluate disconnected quark loops $S_{F}(x, x)$ by solving (1.1) for each lattice site $x$, but it requires prohibitively large computational cost. Instead, we may construct the all-to-all quark propagator, which contains propagations from any lattice site to any site, in an effective way [4]. We determine low-lying eigenvalues $\left\{\lambda_{k}\right\}$ and corresponding eigenvectors $\left\{u_{k}\right\}$ of $D$, and the contribution of these low-modes to the all-to-all quark propagator is calculated exactly as $S_{F, \text { low }}(x, y)=\sum_{k=1}^{N_{e}} \frac{1}{\lambda_{k}} u_{k}(x) u_{k}^{\dagger}(y)$. The number of the low-modes $N_{e}$ is set to 160 (240) on the smaller (larger) lattice. We confirm that low-energy observables, such as disconnected hadron correlators, are well dominated by the low-mode contribution with this choice of $N_{e}$. The small contribution from the higher-modes can be estimated stochastically by the socalled noise method, which is not computationally intensive. This all-to-all propagator is used to calculate disconnected correlators of the flavor-singlet mesons and nucleon. We refer to Refs. [1, $2,6]$ for technical details.

\section{Flavor-singlet meson spectrum}

In the left panel of Fig. 1, we plot vector meson correlators $G_{V, a b}(\Delta t)=\left(1 / N_{t}\right) \sum_{t}\left\langle V_{a}(t+\right.$ $\left.\Delta t) V_{b}^{\dagger}(t)\right\rangle$ of the light $\left(V_{l}=\sqrt{2} \bar{l} \gamma_{k} l\right)$ and strange vector mesons $\left(V_{s}=\bar{s} \gamma_{k} s\right)$ as well as those of the flavor-octet $\left(V_{8}=\sqrt{2 / 3}\left(\bar{l} \gamma_{k} l-\bar{s} \gamma_{k} s\right)\right)$ and singlet mesons $\left(V_{0}=\left(2 \bar{l} \gamma_{k} l+\bar{s} \gamma_{k} s\right) / \sqrt{3}\right)$. The offdiagonal correlators $G_{V,\{l s, s l\}}$ of $V_{l}$ and $V_{s}$ are about two orders of magnitude smaller than the diagonal ones $G_{V,\{l l, s s\}}$, while there is no such large hierarchy between $V_{0}$ and $V_{8}$. We can express $\omega$ and $\phi$ meson states as eigenvectors of the $2 \times 2$ correlator matrices $G_{V, a b}(a, b \in\{l, s\}$ or $\{8,0\})$

$$
\left\{\begin{array}{l}
\phi=1.00(1) V_{s}-0.04(9) V_{l}=0.84(5) V_{8}-0.55(7) V_{0} \\
\omega=0.04(9) V_{s}+1.00(1) V_{l}=0.55(7) V_{8}+0.84(5) V_{0}
\end{array}\right.
$$



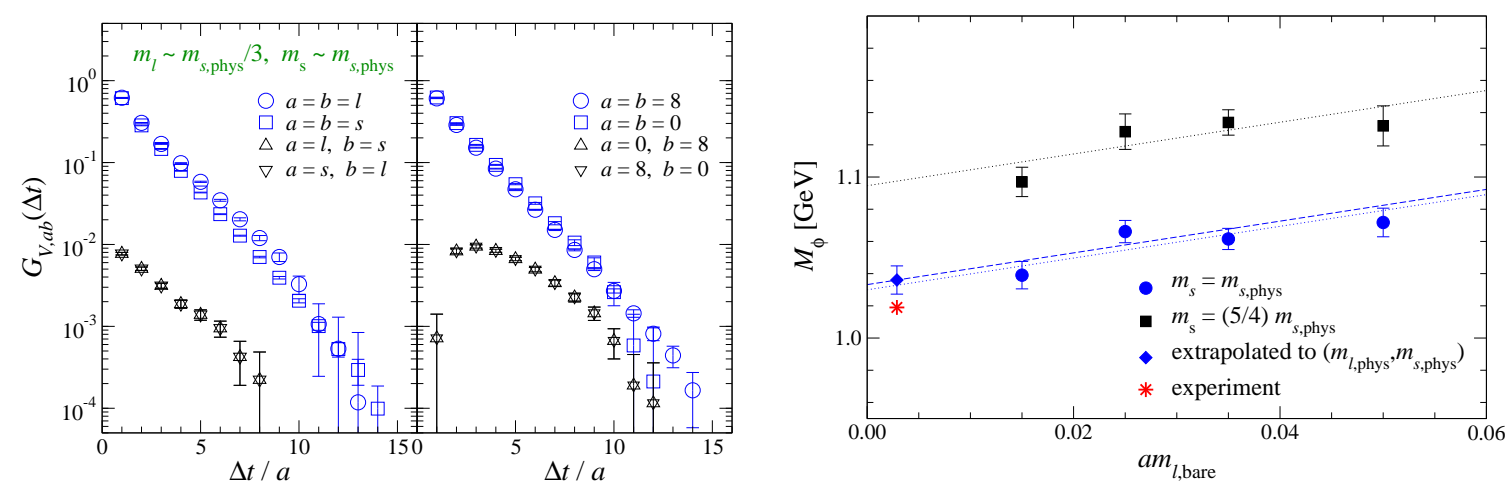

Figure 1: Left panel: vector meson correlators $G_{V, a b}$. Right panel: chiral extrapolation of $M_{\phi}$. The horizontal line represents the bare light quark mass in lattice units, and $m_{s, \text { phys }}$ is 0.081 in this normalization.

These observations imply an almost ideal mixing of the vector mesons, which is expected from the experimental spectrum. The right panel of Fig. 1 shows a linear chiral extrapolation of the mass of the $\phi$ meson state in (2.1). At the physical quark masses, we obtain $M_{\phi}=1036(12)_{\text {stat }}(-91)_{\text {sys }} \mathrm{MeV}$ and $M_{\omega}=854(24)_{\text {stat }}(-92)_{\text {sys }} \mathrm{MeV}$, where the systematic error is estimated by adding higher order terms into the extrapolation form.

A similar analysis for the PS mesons suggests that $\eta$ and $\eta^{\prime}$ mesons are significant mixture of the light $\left(P_{l}\right)$ and strange mesons $\left(P_{s}\right)$ in contrast to the vector mesons:

$$
\eta=0.96(1) P_{l}-0.28(3) P_{s}, \quad \eta^{\prime}=0.28(3) P_{l}-0.96(1) P_{s} .
$$

A linear chiral extrapolation yields $M_{\eta}=620(47)_{\text {stat }} \mathrm{MeV}$ and $M_{\eta^{\prime}}=789(128)_{\text {stat }} \mathrm{MeV}$. The obtained spectrum of the vector and pseudo-scalar mesons is in good agreement with experiment.

\section{Nucleon strange quark content}

We extract the bare value of the strange quark content $\langle N|\bar{s} s| N\rangle_{\text {bare }}$ from the ratio of the threeand two-point functions

$$
R\left(\Delta t, \Delta t^{\prime}\right)=\frac{\sum_{t}\left\langle\mathscr{O}_{N}\left(t+\Delta t+\Delta t^{\prime}\right)\left|\{\bar{s} s(t+\Delta t)\}_{\mathrm{bare}}\right| \overline{\mathscr{O}}_{N}(t)\right\rangle}{\sum_{t}\left\langle\mathscr{O}_{N}\left(t+\Delta t+\Delta t^{\prime}\right) \mid \overline{\mathscr{O}}_{N}(t)\right\rangle} \underset{\Delta t, \Delta t^{\prime} \rightarrow \infty}{\longrightarrow}\langle N|\bar{s} s| N\rangle_{\text {bare }}
$$

A clear non-zero signal shown in the left panel of Fig. 2 is obtained by improving our simulation set up [2]: namely, we use the all-to-all propagator to precisely calculate the disconnected quark loop, and also use smeared nucleon source $\left(\overline{\mathscr{O}}_{N}\right)$ and sink operators $\left(\mathscr{O}_{N}\right)$ in order to reduce excited state contamination of the three- and two-point functions.

In our previous study in two-flavor QCD [2], we observe that the heavy baryon chiral expansion of $\langle N|\bar{s} s| N\rangle_{\text {bare }}$ shows poor convergence at similar quark masses. We therefore employ a simple linear extrapolation to the physical point and also test a constant fit to estimate the systematic uncertainty. Those fits are shown in the right panel of Fig. 2. The extrapolated value is converted to a renormalization group invariant parameter

$$
f_{T_{s}}=\frac{m_{s}\langle N|\bar{s} s| N\rangle}{M_{N}}=0.013(12)_{\text {stat }}(16)_{\text {sys }}
$$



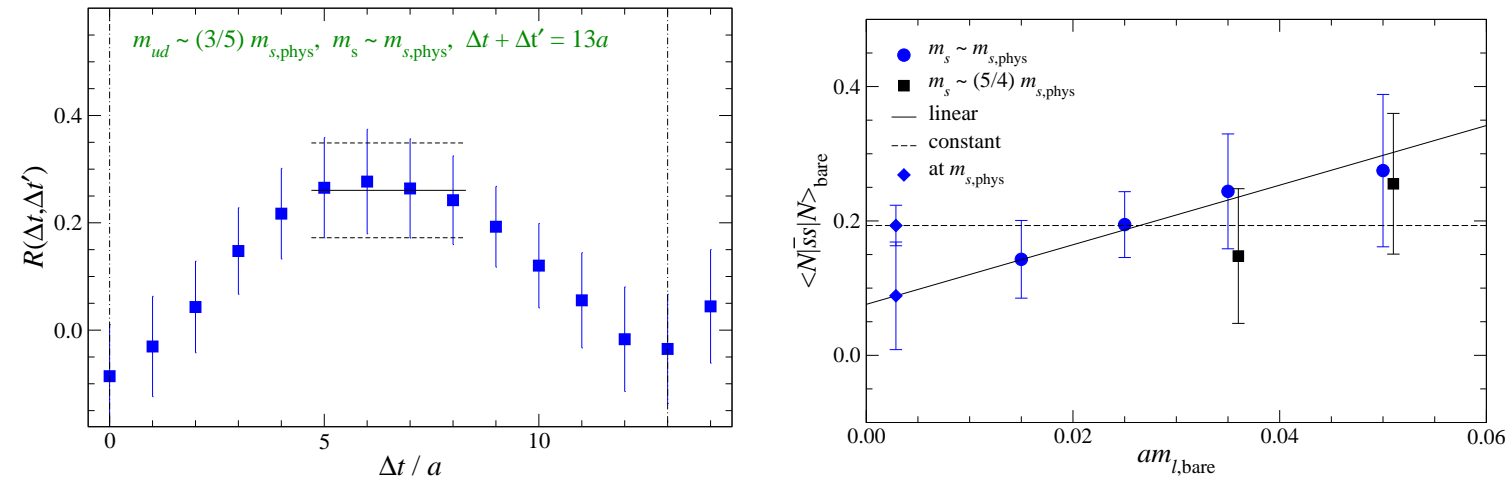

Figure 2: Left panel: ratio $R\left(\Delta t, \Delta t^{\prime}\right)$ with $\Delta t+\Delta t^{\prime}=13 a$ as a function of $\Delta t$. Right panel: chiral extrapolation of $\langle N|\bar{s} s| N\rangle_{\text {bare. }}$. Solid and dashed lines show linear and constant fits at $m_{s}=m_{s, \text { phys. }}$.

We note that chiral symmetry, which is preserved in our simulation, is crucial to avoid a possibly large contamination due to the operator mixing with the light quark content $\langle N|\bar{u} u+\bar{d} d| N\rangle_{\text {bare }}$ [2]. We also emphasize that (3.2) is in good agreement with our previous estimate in two-flavor QCD [2] and our indirect determination through the Feynman-Hellmann theorem [7]: all our studies consistently favor the small strange quark content $f_{T_{s}} \approx 0.01-0.03$.

\section{Conclusion}

In this article, we demonstrated the feasibility of a quantitative study of the flavor-singlet spectrum and nucleon strange quark contents by using the all-to-all propagator. It is interesting to extend this study to other observables, such as the nucleon spin fraction carried by sea quarks.

We also note that the pion scalar form factor has a significant contribution from the disconnected diagram [6]. Although disconnected contributions are generally (much) smaller than connected ones, they can not be naively ignored in future precise determinations of hadron observables.

Numerical simulations are performed on Hitachi SR11000 and IBM System Blue Gene Solution at High Energy Accelerator Research Organization (KEK) under a support of its Large Scale Simulation Program (No. 09/10-09). This work is supported in part by the Grant-in-Aid of the Japanese Ministry of Education, Culture, Sports, Science and Technology (No. 20105005 and 21684013).

\section{References}

[1] T. Kaneko et al. (JLQCD Collaboration), PoS LAT2009, 107 (2009)

[2] K. Takeda et al. (JLQCD Collaboration), arXiv:1011.1964 [hep-lat].

[3] K. Takeda et al. (JLQCD Collaboration), PoS Lattice 2010, 160 (2010).

[4] J. Foley et al. (TrinLat Collaboration), Comput. Phys. Commun, 172, 145 (2005).

[5] H. Matsufuru et al. (JLQCD and TWQCD Collaborations), PoS LATTICE2008, 077 (2008).

[6] S. Aoki et al. (JLQCD and TWQCD Collaborations), Phys. Rev. D 80, 034508 (2009).

[7] H. Ohki et al. (JLQCD Collaboration), Phys. Rev. D 78, 054502 (2008); PoS LAT2009, 124 (2009). 